systolic blood pressure (SBP), and diastolic blood pressure (DBP), and positively correlated with HDL-C However, there is no relation between $25(\mathrm{OH}) \mathrm{D}$ and adiponectin levels among obese children and total sample.

Conclusion Inspite of strong association between vitamin D and adiponectin levels with metabolic risk factors and obesity, there is no relation between $25(\mathrm{OH}) \mathrm{D}$ and adiponectin levels. In obese children, there are significant negative correlations between $25(\mathrm{OH})$ D with lipid profile, and between adiponectin levels with blood pressure. At certain adiponectin level, the relation between it and BMI disappears.

\section{0-053 ADIPONECTIN IS ASSOCIATED WITH METABOLIC SYNDROME IN OBESE TAIWANESE ADOLESCENTS}

${ }^{1} \mathrm{Y}$ Lin, ${ }^{1} \mathrm{P}$ Chang, ${ }^{2} \mathrm{Y}$ Ni. ${ }^{1}$ Pediatrics, Far Eastern Memorial Hospital, New Taipei City, Taiwan; ${ }^{2}$ Pediatrics, National Taiwan University Hospital, Taipei, Taiwan

\subsection{6/archdischild-2014-307384.121}

Background and aims Concurrent with the rise of the incidence in obesity, metabolic syndrome (MS) is increasingly prevalent in obese adolescents. Adiponectin is a major regulator of glucose and lipid homeostasis. Decreased adiponectin levels may be linked to MS. We aimed to test the hypothesis that adiponectin would influence the risk of MS in obese adolescents independent of its insulin sensitizer properties.

Methods This study was a cross-sectional study of the risk factors for MS in obese adolescents. A total of 593 obese subjects aged 10-18 years were recruited. International Diabetes Federation (IDF) consensus was used to define MS. We measured anthropometric, serum biochemical variables, serum adiponectin levels, and biomarkers for insulin resistance. We evaluated the independent influence of the adiponectin level on MS after controlling for the effect of insulin resistance measured by HOMA-IR.

Results 83 (14\%) had MS. Obese adolescents with MS had significantly higher HOMA-IR and lower adiponectin levels than obese adolescents without MS. The adiponectin levels decreased with increasing number of metabolic syndrome components present in obese adolescents $(\mathrm{p}<0.001)$. In multivariate logistic regression analysis, MS was significantly associated with age, body mass index, HOMA-IR, and adiponectin. For every $1 \mu \mathrm{g} /$ $\mathrm{mL}$ decrease in serum adiponectin level, there was an increased risk of having MS with an odds ratio of 1.229 (95\% confidence interval, 1.112 to $1.358, \mathrm{p}<0.001)$.

Conclusions Decreased serum adiponectin level is associated with an increased risk for MS independent of the effects of age, BMI, and insulin resistance in our population of obese Taiwanese adolescents.

\section{0-054 NON-INVAISIVE ASSESSMENT OF LIVER STIFFNESS AND HEPATIC FAT DEPOSITION BY FIBROSCAN IN JAPANESE OBESE CHILDREN}

Y Cho, H Shintaku, D Tokuhara. Pediatrics, Osaka City University Graduate School of Medicine, Osaka, Japan

\subsection{6/archdischild-2014-307384.122}

Backgrounds and aims The aim of our study was to determine the usefulness and feasibility of transient elastography (FibroScan) assessing liver stiffness and hepatic fat deposition in obese children. Methods Obese children (Obese group; BMI-SDS above 90th percentile) and non-obese children without liver disease (Control group) were examined for liver stiffness measurement (LSM) with simultaneous controlled attenuation parameter (CAP) using Fibroscan. LSM and CAP were compared with clinical, biochemical, ultrasound and histological data.

Results Obese group $(\mathrm{n}=40,12.4 \mathrm{y} \pm 3.6 \mathrm{y})$ and the control group ( $\mathrm{n}=78,10.6 \mathrm{y} \pm 4.2 \mathrm{y}$ ) were evaluated for the study. Liver biopsy was performed in 5 patients. The CAP was significantly correlated with ultrasound fatty liver score $(r=0.806, p$ $=0.028)$ and histological steatosis grade $(\mathrm{r}=0.819, \mathrm{p}=$ 0.016). The LSM was significantly correlated with histological fibrosis grade $(\mathrm{r}=0.848, \mathrm{p}=0.005)$. The CAP of the obese group $(293.27 \pm 55.72 \mathrm{~dB} / \mathrm{m})$ showed significantly higher value than control group $(179.45 \pm 44.75 \mathrm{~dB} / \mathrm{m})(\mathrm{p}<0.0001)$. The LSM showed significantly higher value in the obese group compared to the control group $(5.7 \pm 2.3 \mathrm{kPa}$ vs $3.9 \pm 0.9 \mathrm{kPa}, \mathrm{p}<$ 0.0001). In the control group, no biochemical parameters were correlated with LSM or CAP. In the obese group, the LSM correlated to the aspartate aminotransferase $(r=0.694, p<0.0001)$ and the alanine aminotransferase $(\mathrm{r}=0.6748, \mathrm{p}<0.0001)$, whereas the CAP correlated to no parameters.

Conclusions FibroScan is a non-invasive tool to assess the liver stiffness and hepatic fat deposition simultaneously thus useful as a screening tool for nonalcoholic fatty liver disease especially in obese children.

\section{Neonatal Brain and Development Hypoxia - Ischemia}

\section{0-055 FRACTIONAL ANISOTROPY IN WHITE MATTER AND MEAN DIFFUSIVITY IN GREY MATTER CORRELATE TO NEURODEVELOPMENTAL PERFORMANCE FOLLOWING HYPOXIC-ISCHAEMIC ENCEPHALOPATHY}

${ }^{1} \mathrm{~N}$ Tusor, ${ }^{1} \mathrm{~A}$ Makropoulos, ${ }^{1} \mathrm{G}$ Ball, ${ }^{2} \mathrm{~B}$ Bouwen, ${ }^{1} \mathrm{f}$ Allsop, 'D Azzopardi, 'AD Edwards, ${ }^{1} \mathrm{~S}$ Counsell. 'Division of Imaging Sciences and Biomedical Engineering, Department of Perinatal Imaging, Centre for the Developing Brain, King's College London, London, UK; ${ }^{2}$ Department of Neonatology, Erasmus MC Sophia Children's Hospital, Rotterdam, Netherlands

\subsection{6/archdischild-2014-307384.123}

Background and aim Biomarkers are needed to test novel neuroprotective therapies efficiently. The aim was to test the hypothesis that fractional anisotropy (FA) in white matter (WM) and mean diffusivity (MD) in grey matter (GM) correlate to subsequent developmental quotient (DQ) in infants with hypoxicischaemic encephalopathy (HIE).

Methods We studied 40 infants with HIE (median [range] age $\left.39^{+5}\left[36^{+4}-42^{+3}\right]\right)$, who underwent MRI within 21 days of birth and neurodevelopmental assessment at $\geq 12$ months. Infants with a DQ $>2$ SDs below the mean were considered to
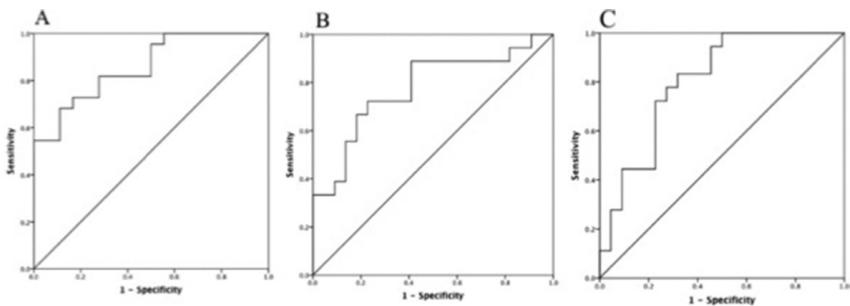

Abstract 0-055 Figure 1 Graphs showing receiver operating characteristic curves to estimate outcome with FA in WM (A), MD in thalami (B) and cortex (C) 